\title{
Measurement of Mixed Biomass Burning and Mineral Dust Aerosol in the Thermal Infrared
}

\author{
C. H. Koehler, E. Lindermeir, T. Trautmann
}

Remote Sensing Technology Institute, German Aerospace Center, Oberpfaffenhofen, Germany

\section{Introduction}

From January 19th to February 6th, 2008, we installed a Fourier transform infrared spectrometer (FTIR) at Praia Airport on the island of Santiago, Cape Verde. Our goal was to measure the combined radiative effect of biomass burning aerosol with mineral dust usually observed here at this time of the year, when mineral dust emerging from the Sahara mixes with biomass burning aerosol transported north-westwards from the Sahelian region. Our measurements were part of the Saharan Mineral Dust Experiment 2 (SAMUM 2) funded by the German Research Foundation (DFG) as continuation of the SAMUM 1 field experiment conducted in Morocco in 2006. SAMUM 2 is a joint venture of several German research institutes and universities and included ground based measurements with spectrometers (visible to thermal infrared), LIDAR and sun photometers. Furthermore samples were taken from the aerosol both at the ground and directly from the aerosol layers with the DLR Falcon research aircraft. We will present our measurements here for the first time along with first results obtained during SAMUM 2.

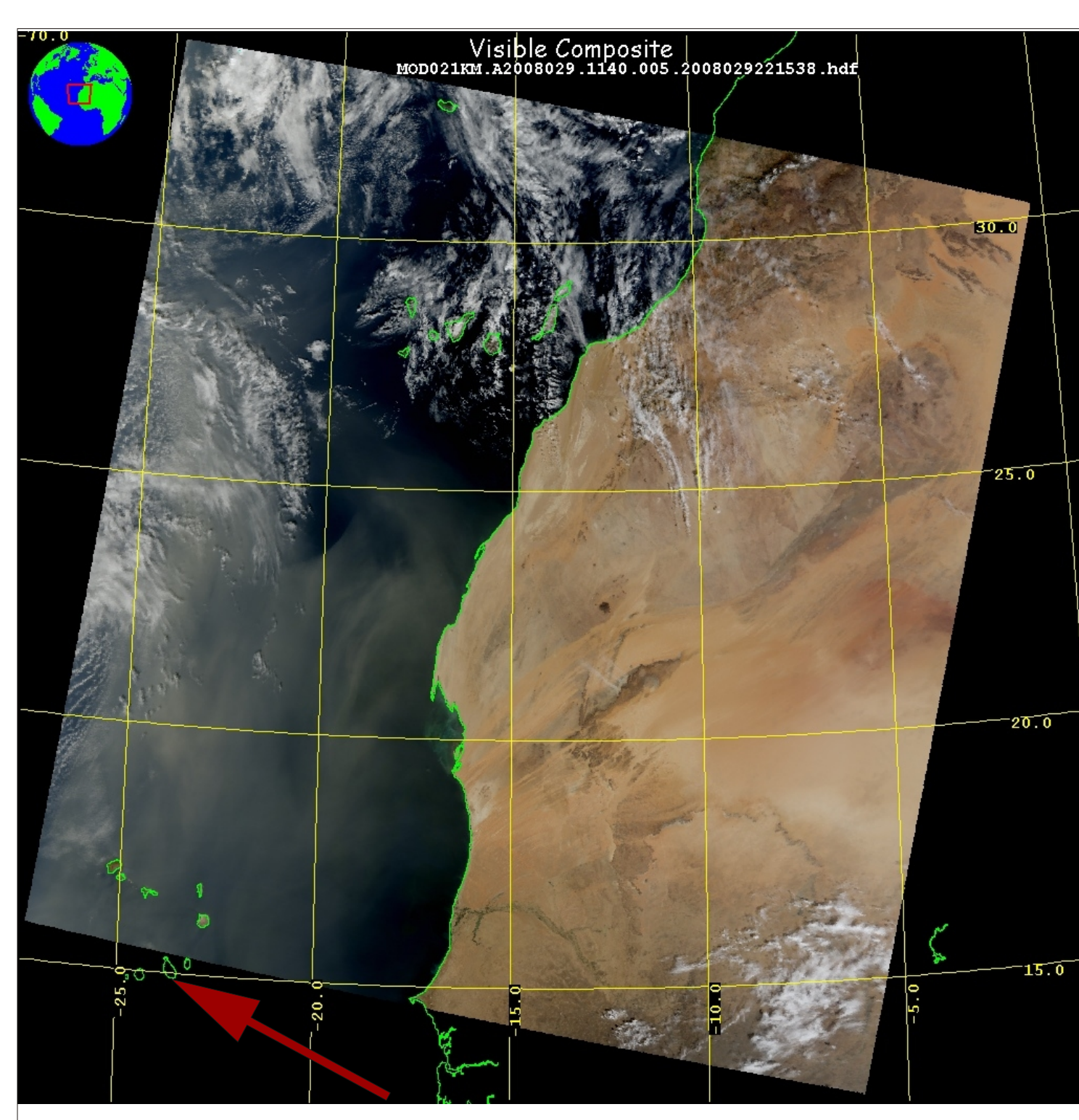

MODIS Image taken January 29 th, 2008 at 11:40
UTC: Mineral dust originating from the Sahara moving westward over the Atlantic and Cap Verde. The red arrow marks Praia Airport, where the instrument was set up.

\section{First Results}

Dust front passage between January 28th and January 29th (see MODIS image on the left):

ine-by-line radiative transfer simulation of clear-sky scenario with radiative transfer code TRAVIS described in [1] including

$\rightarrow$ Pressure, temperature and humidity profiles from radio sonde data

$\rightarrow$ Ozone column density from GOME 2

$\rightarrow$ Remaining data from standard tropical atmosphere

$\rightarrow$ Reduced spectral resolution to $10 \mathrm{~cm}^{-1}$ to smoothen spectra

$\rightarrow$ Calculate brightness temperature difference between measurement and simulation (right)

$\rightarrow$ Significant difference between clear-sky measurements and measurements during dust passage in the atmospheric window region from 800 to $1200 \mathrm{~cm}^{-1}(8-12 \mu \mathrm{m})$

No significant contribution of biomass burning aerosol in the atmospheric window region
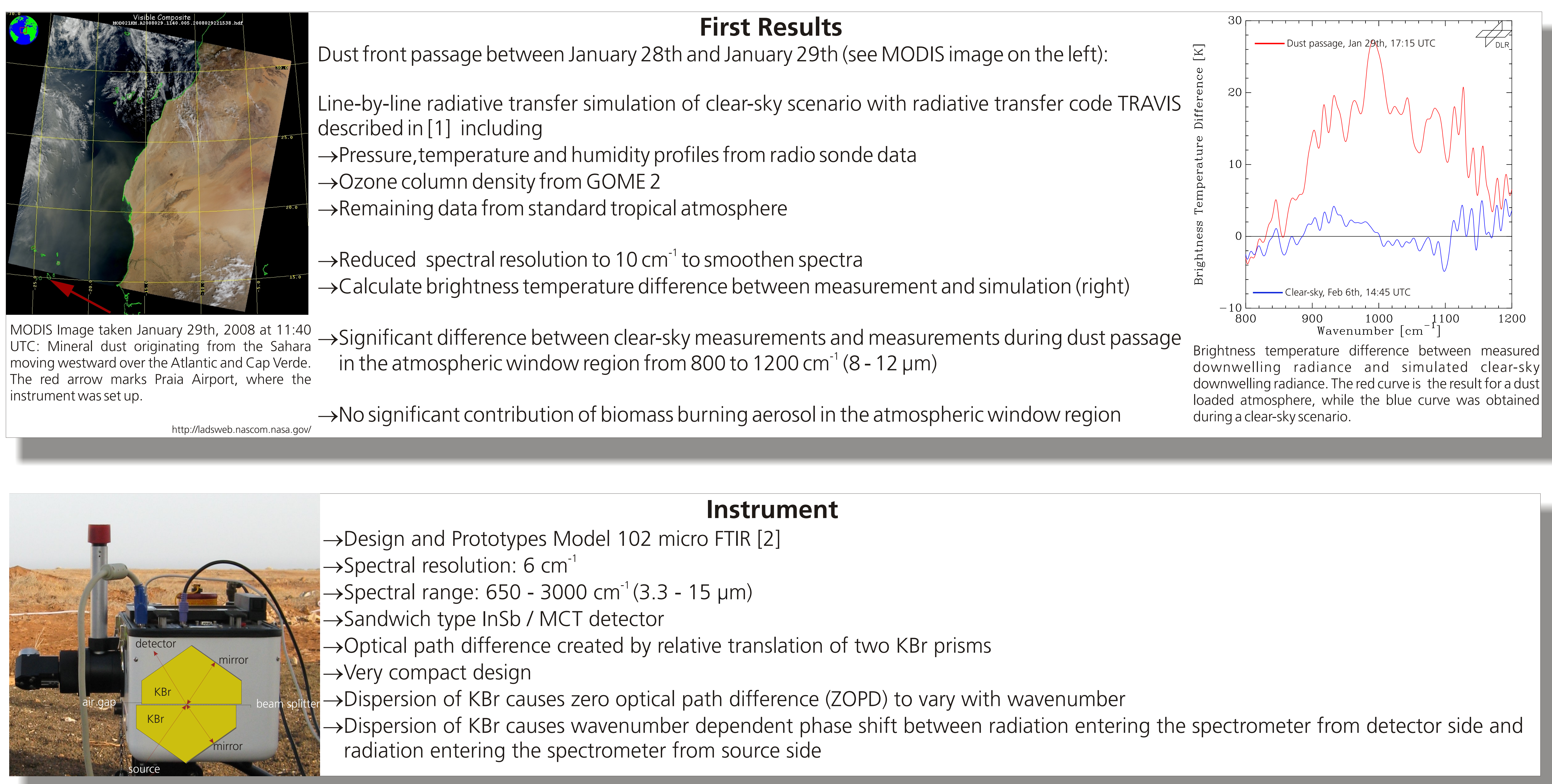

$\rightarrow$ Design and Prototypes Model 102 micro FTIR [2]

\section{Instrument}

$\rightarrow$ Spectral resolution: $6 \mathrm{~cm}^{-1}$

$\rightarrow$ Spectral range: $650-3000 \mathrm{~cm}^{-1}(3.3-15 \mu \mathrm{m})$

$\rightarrow$ Sandwich type InSb / MCT detector

$\rightarrow$ Optical path difference created by relative translation of two $\mathrm{KBr}$ prisms

$\rightarrow$ Very compact design

$\rightarrow$ Dispersion of $\mathrm{KBr}$ causes zero optical path difference (ZOPD) to vary with wavenumber

$\rightarrow$ Dispersion of $\mathrm{KBr}$ causes wavenumber dependent phase shift between radiation entering the spectrometer from detector side and

radiation entering the spectrometer from source side

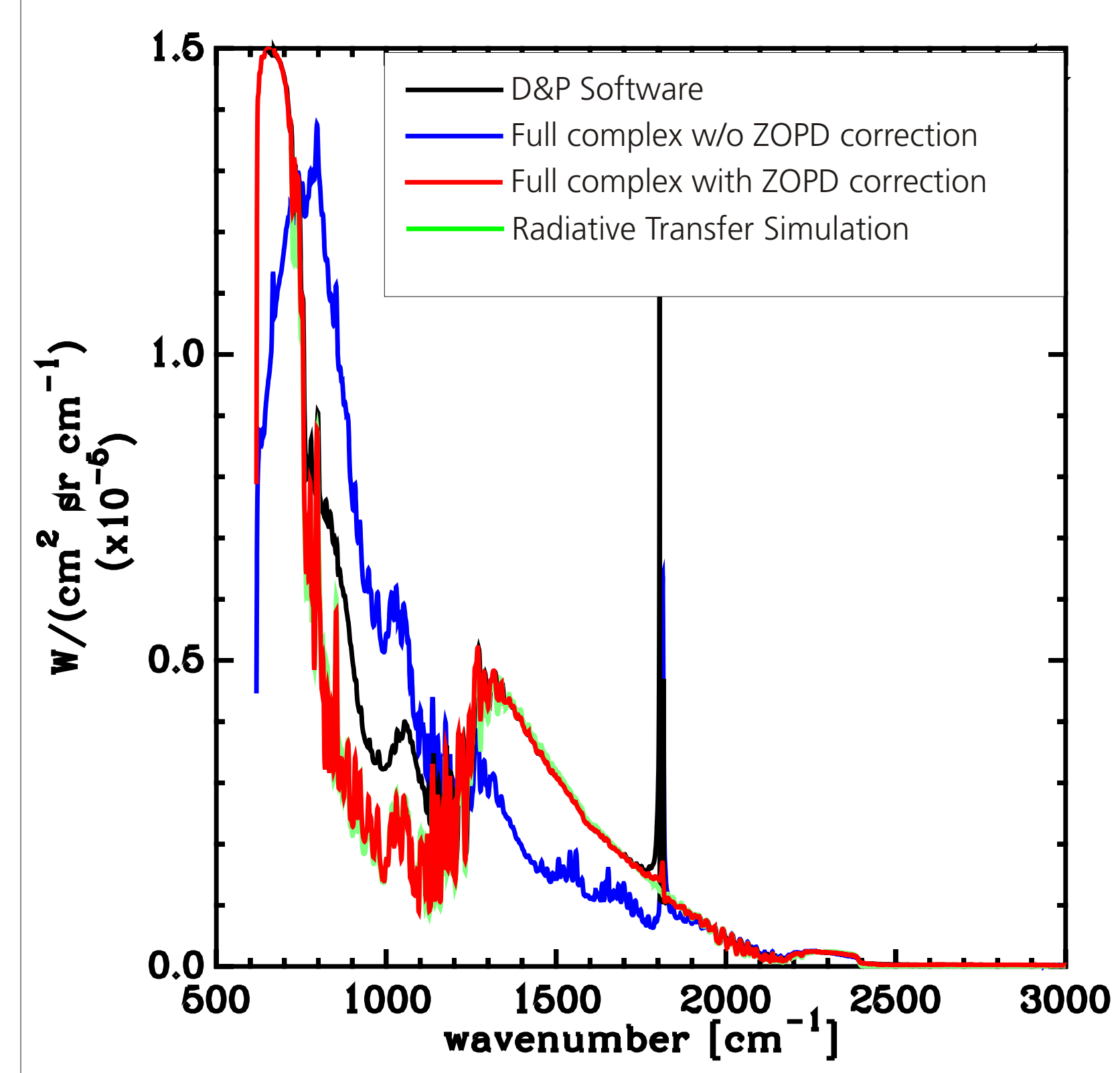

\section{Calibration}

In order to improve the results for spectra with large dynamic range, we use the following calibration procedure:

$\rightarrow$ Create broadband interferogram with FIR digital filter $600-3000 \mathrm{~cm} \Rightarrow$ remove noise

$\rightarrow$ Create narrow-band interferogram with FIR filter $1300-1600 \mathrm{~cm} \Rightarrow$ reduce influence of dispersion

$\rightarrow$ Set ZOPD of broadband interferogram to center burst of narrow-band interferogram

$\rightarrow$ Calibration method described by Revercomb [3] with complex-valued instrument radiance and

complex-valued instrument response

$\rightarrow$ Use 3 black body temperatures at $3^{\circ} \mathrm{C}, 13^{\circ} \mathrm{C}$ and $23^{\circ} \mathrm{C}$ instead of the usual 2 as described by Lindermeir [4]

$\rightarrow$ Downwelling radiances agree well with radiative transfer simulation compared to other calibration methods (left)

A comparison of radiance spectra obtained via differen calibration methods and a line-by-line radiative transfer obtained from the instrument software (black) and the full complex calibration without ZOPD correction via narrow-band filtering (blue) lead to considerable deviation in the resulting spectra. In contrast to that, the radiance obtained through full complex calbration for

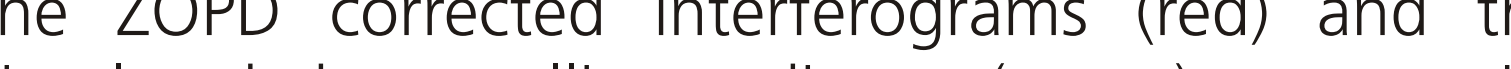
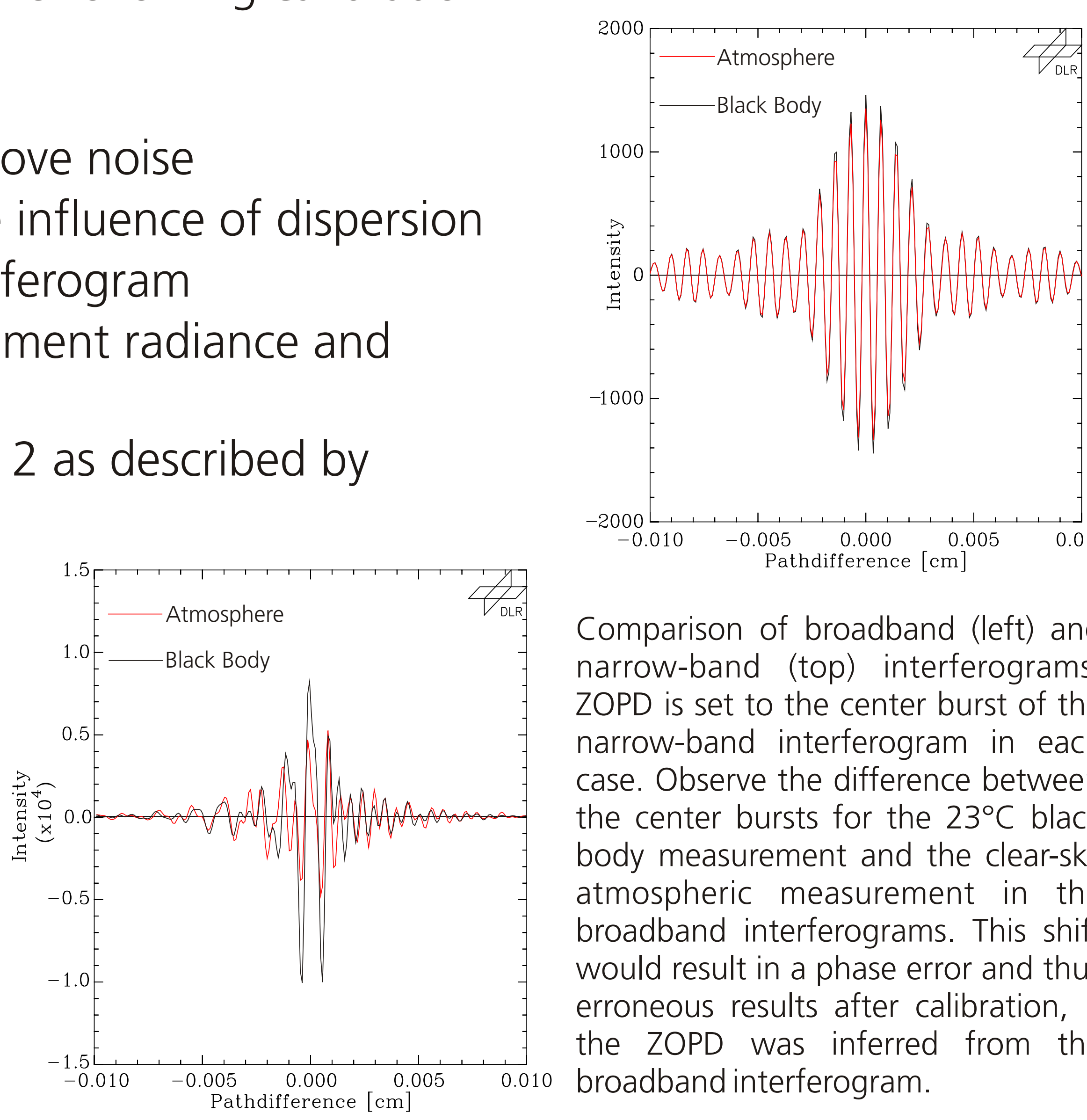

Comparison of broadband (left) and ZOPD is set to the center burst of the narrow-band interferogram in each the center bursts for the $23^{\circ} \mathrm{C}$ black body measurement and the clear-sky atmospheric measurement in the broadband interferograms. This shift would result in a phase error and thus erroneous results after calibration,

\section{References}

11. Otto et al, "Solar radiative effects of a Saharan dust plume observed during SAMUM assuming spheroidal model particles", Tellus, 61B, 2009, accepted

[2] A. R. Korb, P. Dybward, W. Wadsworth, J. Salisbury," Portable Fourier transform infrared spectroradiometer for field measurements of radiance and emissivity", Applied Optics, Vol. 35, No. 10, 1 April 1996 3. H. E. Revercomb et al, "Radiometric calibration of IR Fourier transtorm spectrometers: solution to a problem with the High-Resolution Interferometer Sounder", Applied
4] E. Lindermeir et al, "Calibration of a Fourier transform spectrometer using three blackbody sources", Applied Optics, Vol. 31, No. 22,1 August 1992 